\title{
Head CT: Image quality improvement of posterior fossa and radiation dose reduction with ASiR - comparative studies of CT head examinations
}

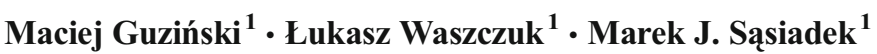

Received: 14 August 2015 /Revised: 11 December 2015 / Accepted: 18 December 2015 / Published online: 23 January 2016

(C) The Author(s) 2016. This article is published with open access at Springerlink.com

\begin{abstract}
Objectives To evaluate head CT protocol developed to improve visibility of the brainstem and cerebellum, lower bone-related artefacts in the posterior fossa and maintain patient radioprotection.

Methods A paired comparison of head CT performed without Adaptive Statistical Iterative Reconstruction (ASiR) and a clinically indicated follow-up with $40 \%$ ASiR was acquired in one group of 55 patients. Patients were scanned in the axial mode with different scanner settings for the brain and the posterior fossa. Objective image quality analysis was performed with signal-to-noise ratio (SNR) and contrast-tonoise ratio (CNR). Subjective image quality analysis was based on brain structure visibility and evaluation of the artefacts.

Results We achieved $19 \%$ reduction of total DLP and significantly better image quality of posterior fossa structures. SNR for white and grey matter in the cerebellum were $34 \%$ to $36 \%$ higher, respectively, CNR was improved by $142 \%$ and subjective analyses were better for images with ASiR.

Conclusions When imaging parameters are set independently for the brain and the posterior fossa imaging, $\mathrm{ASiR}$ has a great potential to improve CT performance: image quality of the brainstem and cerebellum is
\end{abstract}

Łukasz Waszczuk

lukasz.waszczuk@reagan.com

1 Department of General Radiology, Interventional Radiology and Neuroradiology, Wroclaw Medical University, Borowska 213, 50-556 Wrocław, Poland improved, and radiation dose for the brain as well as total radiation dose are reduced.

Key Points

-With ASiR it is possible to lower radiation dose or improve image quality

-Sequentional imaging allows setting scan parameters for brain and posterior-fossa independently

-We improved visibility of brainstem structures and decreased radiation dose

- Total radiation dose (DLP) was decreased by $19 \%$

Keywords Brain diseases/radiography $\cdot$ Radiation dosage · Radiation protection methods · Computer-assisted methods . Tomography, X-ray computed methods

\section{Introduction}

From the introduction of computed tomography (CT) into clinical practice in 1972, the filtered back projection (FBP) algorithm has been used for image reconstruction [1]. Within the next few decades, many advances in CT technology were made. Simultaneously the number of indications for $\mathrm{CT}$ has markedly increased. Widening the scope of CT application has resulted in an increase in the number of $\mathrm{CT}$ examinations performed each year and thus in higher population doses from medical sources [2]. On the other hand, widespread interest in radiation protection has increased with the aim of lowering the possible hazards associated with radiation exposure $[3,4]$. For this reason several CT protocols have been developed that aimed to lower radiation dose. Many of them are based on modification of scanning parameters and decreasing the tube current-time product as well as lowering tube voltage. 
In recent years the Adaptive Statistical Iterative Reconstruction (ASiR, GE Healthcare, Milwaukee, WI, USA) method has been introduced into CT. The final image is the result of multiple repeated steps in which the image quality is improved with statistical methods. Previous studies have shown that ASiR improves the image quality in abdominal $\mathrm{CT}$ and has a potential for a reduction in radiation dose while maintaining diagnostic acceptability $[5,6]$.

Earlier studies on ASiR in head CT showed similar results [7-10]. However, to our knowledge all of them were performed in two distinct groups of patients. In our study we compared ASiR and FBP protocols in one group of patients who underwent head CT twice for clinically indicated purposes.

Another purpose of this study was to better fit scanning protocols to the posterior fossa structures in order to decrease the scatter noise and the beam hardening artefacts. We performed head CT scanning in the axial mode with different scanning parameters for the brain and the posterior fossa while previous studies have reported on helical scanning mode with one scanner setting for the whole head. Thus, we also aimed to improve the brainstem and the posterior fossa visibility without increasing exposure to the brain. To our knowledge, previously published papers did not describe the impact of ASiR on the posterior fossa imaging.

\section{Methods and materials}

\section{Study group}

In this Institutional Review Board approved study we retrospectively examined patients who underwent two clinically indicated head CTs during one period of hospitalization. We reviewed 355 consecutive patients who underwent head CT in the Emergency Department. We included patients who underwent clinically indicated follow-up head CT. The exclusion criteria were: (1) neurosurgical treatment (clipping, coiling, drainage, etc.), (2) motion artefacts and (3) mass effect (severe haematoma, oedema, neoplasms, etc.). Exclusion criteria were carefully evaluated in order to avoid image quality degradation caused by artefacts from foreign bodies, surgery and severe disturbances in brain morphology.

The first CT examination was performed in the Emergency Department with a VCT Lightspeed, 64-row scanner (GE Healthcare) with FBP reconstruction. The subsequent head CT was performed with a Discovery $750 \mathrm{HD}$ (GE Healthcare) and images were reconstructed with $40 \% \mathrm{ASiR}$.

Scanning was performed in sequential mode. All patients were positioned supine; tube voltage was $120 \mathrm{kVp}$; beam collimation was at $2.5 \mathrm{~mm}$ for the posterior fossa and $5 \mathrm{~mm}$ for the cerebrum; scan range was $14 \pm 1 \mathrm{~cm}$; scan rotation time was $1 \mathrm{~s}$. Automatic exposure control (AEC) was activated.
The noise index (NI) was increased from 2.8 to 4.0 for ASiR and the maximum $\mathrm{mA}$ increased from 303 in the initial examination to 320 in the follow-up ASiR examination. Radiation dose descriptors were derived from the dose report automatically stored in PACS (Picture Archiving and Communication Systems). CT dose index volume (CTDI ${ }_{\mathrm{vol}}$ ), dose length product (DLP) and scan range were recorded for both the FBP and ASiR groups.

\section{Subjective image quality}

Image pairs were reviewed independently by two radiologists with 8 and 10 years' experience, respectively, in neuroradiology. The readers were blinded to the scanning protocols. Images were displayed in the brain window $(80 \mathrm{~W}, 40 \mathrm{~L})$ on an Advantage Workstation AW 4.6 (GE Healthcare). In the subjective image quality assessment the image noise, posterior fossa artefacts and diagnostic acceptability were taken into account.

The readers were instructed to evaluate the image sharpness, the grey and white matter differentiation, and the visibility of the basal ganglia, pons, cerebellum and ventricular system. Images of excellent quality were graded 3; images of good quality were graded 2; images of poor quality but adequate for evaluation were graded 1. The image quality assessment was done according to European Guidelines on Quality Criteria for Computed Tomography [11].

\section{Objective image quality}

In the objective image quality assessment, the signal-to-noise (SNR) and contrast-to-noise (CNR) ratios were analysed. For each data-set five regions-of-interest (ROIs) were manually positioned. The ROI area varied from $20-30 \mathrm{~mm}^{2}$ to encompass homogeneity of measured tissues. The first two ROIs were set in the white matter of the posterior limb of the internal capsule and grey matter of the caudate nucleus. The other two ROIs were set in the white and grey matter of the cerebellum. The fifth ROI was drawn in cerebrospinal fluid of the fourth ventricle (Figs. 1 and 2). The mean attenuation value (mean) within the ROI and the standard deviation (SD) were considered for signal level and noise.

The SNR was then calculated as the mean divided by the $\mathrm{SD}$. The CNR was calculated for ROI pairs of white and grey matter with the following formula where the difference in means are divided by the square root of the sum of the squared noise in two ROIs:

$\mathrm{CNR}=\frac{\text { Mean1 }- \text { Mean2 }}{\sqrt{\mathrm{SD}^{2}+\mathrm{SD}^{2}}}$ 


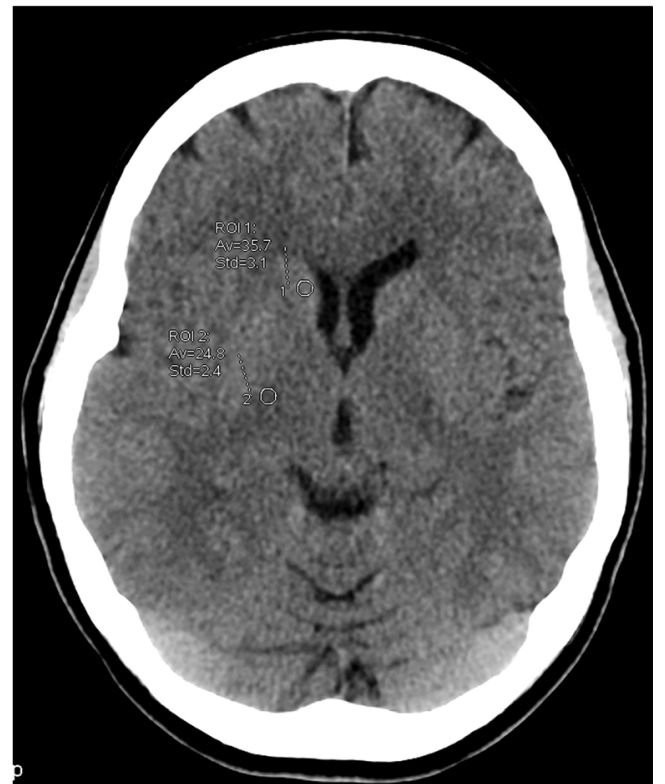

Fig. 1 Small regions of interest positioning in the white matter of the posterior limb of the internal capsule and grey matter of the caudate nucleus

\section{Statistics}

Statistical analysis was performed using R Statistical Software (R Foundation for Statistical Computing, Vienna, Austria) [12].

Differences in SNR and CNR measurements between image pairs were tested using the paired t-test with Welsh modification for unequal variances. To reduce the possibility of significance due to chance because of multiple statistical

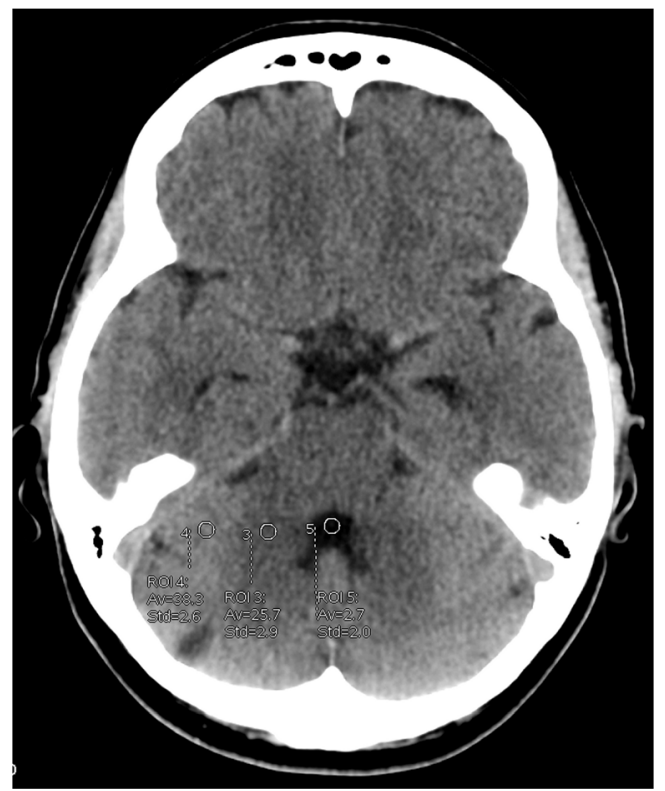

Fig. 2 Small regions of interest positioning in the posterior fossa, in the white and grey matter of the cerebellum and the fifth in the cerebrospinal fluid of the fourth ventricle
Table 1 Objective image quality assessment of head CT: Adaptive Statistical Iterative Reconstruction (ASiR) compared to filtered back projection (FBP)

\begin{tabular}{|c|c|c|c|c|}
\hline & ASiR & FBP & ASiR vs.FBP & p-value \\
\hline \multicolumn{5}{|l|}{ Cerebrum } \\
\hline SNR GM & $11.6 \pm 2.8$ & $9.7 \pm 1.9$ & $+20 \%$ & NS \\
\hline SNR WM & $8.7 \pm 2.5$ & $7.3 \pm 1.7$ & $+19 \%$ & NS \\
\hline CNR & $0.12 \pm 0.09$ & $0.08 \pm 0.05$ & $+50 \%$ & NS \\
\hline \multicolumn{5}{|c|}{ Posterior fossa } \\
\hline SNR GM & $13.5 \pm 3.6$ & $9.9 \pm 2.5$ & $+36 \%$ & $<0.0001$ \\
\hline SNR WM & $9.5 \pm 2.9$ & $7.1 \pm 1.7$ & $+34 \%$ & $<0.0001$ \\
\hline CNR & $0.17 \pm 0.10$ & $0.07 \pm 0.04$ & $+142 \%$ & $<0.0001$ \\
\hline \multicolumn{5}{|c|}{ Fourth ventricle } \\
\hline SNR & $1.7 \pm 0.8$ & $1.4 \pm 0.7$ & $+21 \%$ & NS \\
\hline
\end{tabular}

Values are given as mean $\pm \mathrm{SD}$

$S N R$ signal-to-noise ratio, $C N R$ contrast-to-noise ratio, $G M$ grey matter, $W M$ white matter

testing, the Bonferroni correction was applied to the p-value. Significance was assumed only when the $p$-value was less than 0.01 .

The paired t-test was used to compare subjective image quality assessments between image pairs with a 0.05 level of significance.

\section{Results}

We included in the study 55 patients who underwent the head CT twice: once each with ASiR and FBP. Using ASiR we

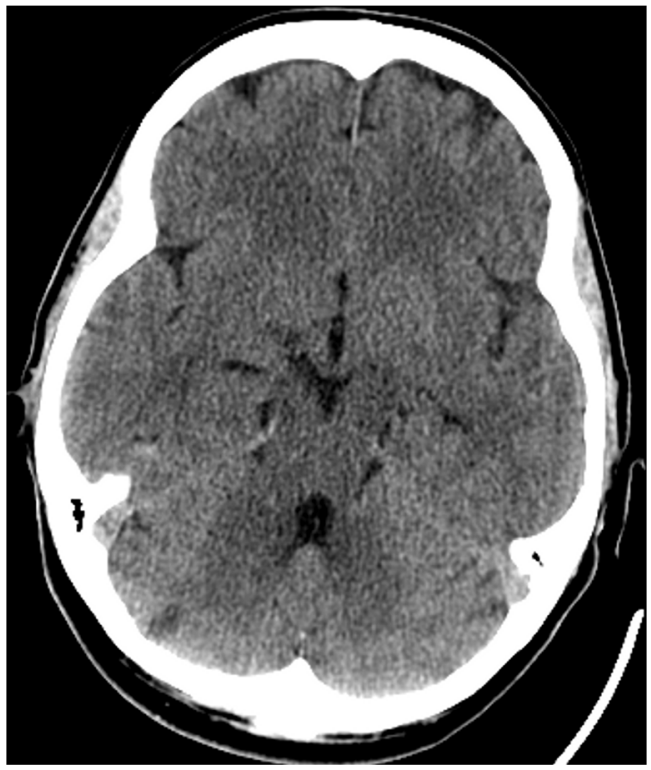

Fig. 3 CT scan of a 56-year-old patient after a minor head trauma. Initial examination filtered back projection (FBP) reconstruction algorithm axial image of the posterior fossa with acceptable image quality 


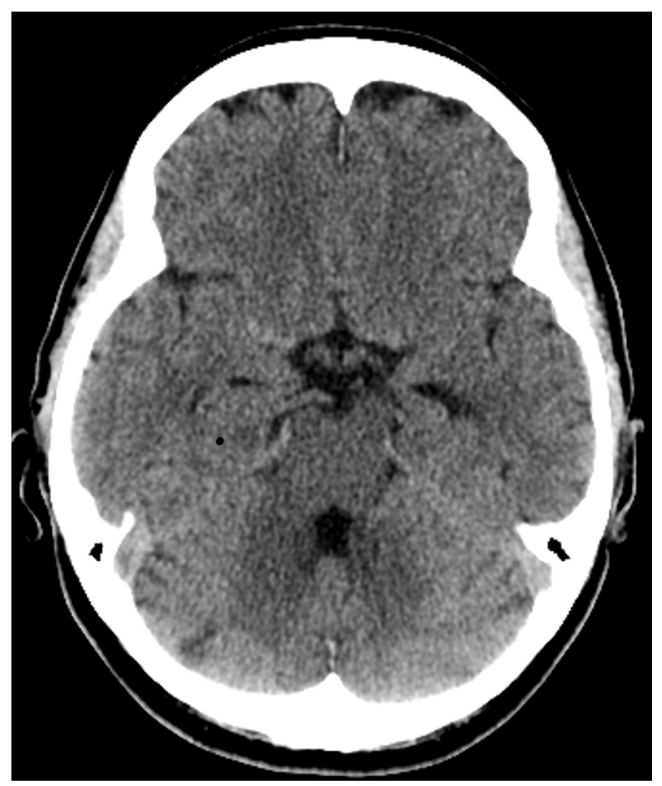

Fig. 4 Follow-up examination in the same patient with $40 \%$ Adaptive Statistical Iterative Reconstruction (ASiR) and overall dose reduction with acceptable image quality but slightly lower noise in the posterior fossa

achieved a statistically significant improvement in posterior fossa imaging. In the objective image quality analysis the SNR of both white and grey matter in the cerebellum was $34 \%$ and $36 \%$ higher. We also achieved a $142 \%$ increase in the CNR of the posterior fossa. All SNR and CNR measurements for the brain and the posterior fossa are presented in Table 1 . In the subjective image quality analysis two researchers reported a slightly better quality of ASiR images, but there was no significant difference in overall subjective image quality assessment: 2.7 versus 2.8 for the ASiR protocol for the first observer and 2.6 versus 2.8 for the second observer (Figs. 3 and 4).

In the ASiR protocol we achieved a $30 \%$ decrease in radiation dose (DLP) for the brain, and a $19 \%$ decrease in overall radiation dose from head $\mathrm{CT}$, whereas the scan range in the two cohorts did not differ significantly. The DLP for the posterior fossa in the ASiR protocol increased by $9 \%$. CTDI ${ }_{\mathrm{vol}}$ in the brain and in the posterior fossa were 36.2 and $59.7 \mathrm{mGy}$, respectively. Radiation dose descriptors for ASiR and FBP protocols are shown in Table 2.

Attenuation value of white and grey matter and cerebrospinal fluid remained at the same level in both cohorts; no statistically significant difference was noted. These results are presented in Table 3.

\section{Discussion}

In our study we investigated head $\mathrm{CT}$ in terms of an image reconstruction algorithm (i.e. ASiR and FBP protocols) as well as radiation dose to the brain and the posterior fossa. The advantage of this study was that we gathered paired sets of ASiR and FBP examinations from one group of patients and we were able to perform paired comparisons of the acquired images. To our knowledge all other published studies compared ASiR and non-ASiR CT protocols in two separate groups of patients and thus might have been confounded by differences in the subjects' brain morphology [7-10].

As ASiR application optimises noise in the reconstructed images [13], it may serve two general purposes: (1) to lower radiation dose without compromising image quality and (2) to improve the image quality maintaining radiation dose. This first approach, i.e. reducing the radiation dose with ASiR, is applied in the majority of published papers. However, we decided to scan the posterior fossa with ASiR and the same radiation dose (DLP) as in the previous protocol with FBP in order to improve the image quality of the posterior fossa structures. For this reason we performed head CT in which the brain and the posterior fossa are scanned with different scanner settings. As a result, with the introduction of ASiR we maintained the radiation-dose level in the posterior fossa and we achieved a 34-36\% increase in SNR in white and grey matter, and a $142 \%$ increase in CNR. Our results have clinical importance because images of the cerebellum, pons and medulla were always compromised by artefacts resulting from skull base bones [14, 15].
Table 2 Radiation dose in head CT: Adaptive Statistical Iterative Reconstruction (ASiR) compared to filtered back projection (FBP)

\begin{tabular}{lllll}
\hline & ASiR & FBP & ASiR vs. FBP & p-value \\
\hline DLP (mGy*cm) & & & & \\
$\quad$ Overall & $654.5 \pm 77.0$ & $804.2 \pm 52.3$ & $-19 \%$ & $<0.0001$ \\
Cerebrum & $390.3 \pm 75.5$ & $560.6 \pm 51.8$ & $-30 \%$ & $<0.0001$ \\
Posterior fossa & $264.2 \pm 31.9$ & $243.6 \pm 5.5$ & $+8 \%$ & $<0.0001$ \\
CTDI $_{\text {vol }}(\mathrm{mGy})$ & & & $-30 \%$ & $<0.0001$ \\
Cerebrum & $36.2 \pm 4.5$ & $51.5 \pm 3.0$ & $+7 \%$ & $<0.0001$ \\
Posterior fossa & $59.7 \pm 3.7$ & $55.9 \pm 2.5$ & $+2 \%$ & $\mathrm{NS}$ \\
Scan range $(\mathrm{cm})$ & $14.3 \pm 1.1$ & $14.6 \pm 1.1$ & & \\
\hline
\end{tabular}

$D L P$ dose-length product, $C T D I_{v o l}$ volumetric CT dose index 
Table 3 Attenuation value in head CT: Adaptive Statistical Iterative Reconstruction (ASiR) compared to filtered back projection (FBP)

\begin{tabular}{llll}
\hline & ASiR & FBP & p-value \\
\hline Cerebrum & & & \\
$\quad$ Attenuation GM & $32.6 \pm 3.0$ & $32.5 \pm 2.4$ & NS \\
$\quad$ Attenuation WM & $23.8 \pm 1.7$ & $24.1 \pm 1.8$ & NS \\
Posterior fossa & & & \\
$\quad$ Attenuation GM & $39.2 \pm 2.7$ & $38.6 \pm 3.2$ & NS \\
$\quad$ Attenuation WM & $26.2 \pm 2.3$ & $27.5 \pm 2.8$ & NS \\
Fourth ventricle & & & \\
$\quad$ Attenuation CSF & $3.9 \pm 1.8$ & $5.4 \pm 2.6$ & $\mathrm{NS}$ \\
\hline
\end{tabular}

In our study the mean $\mathrm{CTDI}_{\mathrm{vol}}$ to the brain and the posterior fossa were 36.2 and $59.7 \mathrm{mGy}$, respectively, and were well below the routine diagnostic reference head level which is 75 mGy [16]. For comparison (Table 4) the head CT protocol designed by the American Association of Physicists in Medicine in the same GE Discovery scanner as used in our institution has a CTDI ${ }_{\mathrm{vol}}$ for brain and posterior fossa of 41.9 and 69.7 mGy, respectively [16].

The mean DLP in our study was $654 \mathrm{mGy} * \mathrm{~cm}$, whereas in other studies on ASiR and non-ASiR protocols of Kilic et al. [7], Rapalino et al. [8] and Komlosi et al. [10], DLP was 748, 932 and 1,191 $\mathrm{mGy}^{*} \mathrm{~cm}$, respectively. On the other hand, Ren et al. [17] reported a mean DLP of routine head $559 \mathrm{mGy} * \mathrm{~cm}$ that was $15 \%$ lower than in our study. However, in Ren et al.'s study the posterior fossa was scanned in helical mode with $\mathrm{CTDI}_{\mathrm{vol}} 38.1$, whereas in our study the CTDI $\mathrm{vol}_{\text {of }}$ of the posterior fossa was $59.2 \mathrm{mGy}$. This is very important for image quality of the brainstem and cerebellum because as the radiation dose decreases, the images are noisier and more susceptible to artefacts.

It should be emphasized that the head CT protocol in the axial mode employed for a routine head CT examination in our institution allows us to scan the brain with a lower $\mathrm{CTDI}_{\mathrm{vol}}$ and a lower current time product $\left(\mathrm{mA}^{*} \mathrm{~s}\right)$ as compared to that for the posterior fossa slices. It is important because in helical mode the whole head is scanned in the same mAs settings, thus it should be a compromise between the brain and posterior fossa, where the cerebellum and the brainstem may be degraded by bone related artefacts. Kilic et al. [7] investigated an axial head protocol with $\mathrm{CTDI}_{\mathrm{vol}}$ of the posterior fossa of $93.5 \mathrm{mGy}$ and could have achieved posterior fossa slices of better quality than in our study. In the studies of Rapalino et al. [8] and Komlosi et al. [9] the heads were scanned in helical mode with $\mathrm{CTDI}_{\mathrm{vol}}$ values similar to our study, but using helical mode and constant scanner settings for the brain and posterior fossa they achieved a total DLP 1.5-2 times greater.

With the axial scanning we also avoided the over-ranging phenomenon associated with helical scanning. In helical scan mode every reconstructed section is obtained with interpolation of projection data from neighbouring rotations, thus irradiation of neighbouring tissues is inevitable in helical scanning [18-20].

In order to reduce eye lens exposure, we set the scanned volume border at the line between the supraorbital ridge and posterior margin of the foramen magnum [16]. Precise gantry angulation has an additional advantage. In posterior fossa imaging the beam hardening artefacts have their origin in the relatively large amount of bones in the base of the skull that are thicker than bones that surround the brain. When the margin of the scanned volume is set to the basis of the occipital bone, the lowest imaged slices of cerebellum are adjacent and parallel to the skull base. As a consequence, the beam hardening effects of the $x$-ray beam are reduced and the number of bony artefacts is decreased.

In our institution ASiR was introduced with a new high definition scanner with a unique Gemstone detector (GD). Thus, we are not able to assess how reconstruction algorithm
Table 4 Comparison of radiation-dose from head $\mathrm{CT}$ examinations with Adaptive Statistical Iterative Reconstruction (ASiR) image reconstruction

\begin{tabular}{lllll}
\hline Head CT protocols with ASiR & & & \\
& Scan mode & \multicolumn{2}{l}{ CTDIvol } & Total DLP \\
\cline { 3 - 4 } & & Posterior fossa & Brain & \\
\hline Our study & Axial & 59.2 & 36.4 & 654.5 \\
Kilic et al. 2011 [7] & Axial & 93.5 & 38.6 & 748.5 \\
Ren et al. 2012 [17] & Helical & 38.1 & & 559.0 \\
Rapalino et al. 2012 [8] & Helical & 49.7 & & 932.2 \\
Komlosi et al. 2014 [9] & Helical & 57.1 & & $1,190.9$ \\
AAPM 2012 ${ }^{1}[16]$ & Axial & 69.7 & 41.9 & Not provided \\
& Helical & 38.4 & & Not provided \\
\hline
\end{tabular}

\footnotetext{
${ }^{1}$ The collection of routine head CT protocols by the American Association of Physicists in Medicine for different scanner vendors and models. Radiation doses for GE Discovery $750 \mathrm{HD}$ with are ASiR provided here.
}

CTDIvol volume CT dose index, DLP dose length product 
and detector technology contribute to the dose reduction and image quality improvement that we achieved. As GD has a better performance compared to the older detectors (e.g. the afterglow time is significantly shorter), we think that using the new detector influenced the results.

Another limitation is that in objective image quality assessment we used SNR and CNR values that were questioned by some authors, because IR algorithms gain SNR optimisation [21]. However, this approach for the image quality assessment is widely used in other studies that evaluate iterative reconstruction algorithms and model-based techniques [10, 22-24].

In conclusion, in our study we found advantages in using ASiR in head CT protocols. Axial mode provides the possibility of adjusting scanning parameters for the brain and posterior fossa. With the new protocol we improved image quality of the brainstem and cerebellum as well as reducing the total radiation dose. This scanning protocol has been introduced in our Institution for routine head CT. However, head CT protocols require a thorough evaluation in terms of scanning mode (axial vs. helical), reconstruction algorithm, current time product (mAs) and radiation dose delivered (CTDI $\mathrm{vol}_{\mathrm{v}}$ and DLP). Further studies are needed in this field.

Acknowledgments The scientific guarantor of this publication is Maciej Guziński. The authors of this manuscript declare no relationships with any companies whose products or services may be related to the subject matter of the article. The authors state that this work has not received any funding. One of the authors has significant statistical expertise. Institutional Review Board approval was obtained. Written informed consent was waived by the Institutional Review Board. Some study subjects or cohorts have been previously reported in an oral presentation at ECR 2014, B-0935. Methodology: retrospective, case-control study, performed at one institution.

Open Access This article is distributed under the terms of the Creative Commons Attribution-NonCommercial 4.0 International License (http://creativecommons.org/licenses/by-nc/4.0/), which permits any noncommercial use, distribution, and reproduction in any medium, provided you give appropriate credit to the original author(s) and the source, provide a link to the Creative Commons license, and indicate if changes were made.

\section{References}

1. Kak AC, Slaney M (2001) Principles of Computerized Tomographic Imaging. 2nd edn. Society of Industrial and Applied Mathematics. Society of Industrial and Applied Mathematics, http://www.slaney.org/pct/pct-toc.html

2. Mettler FA Jr, Bhargavan M, Faulkner K et al (2009) Radiologic and nuclear medicine studies in the United States and worldwide: frequency, radiation dose, and comparison with other radiation sources: 1950-2007. Radiology 253:520-531

3. Brenner DJ, Elliston CD, Hall EJ, Berdon WE (2001) Estimated risks of radiation-induced fatal cancer from pediatric CT. AJR Am J Roentgenol 176:289-296

4. Pearce MS, Salotti JA, Little MP et al (2012) Radiation exposure from CT scans in childhood and subsequent risk of leukaemia and brain tumours: a retrospective cohort study. Lancet 380:499-505
5. Silva AC, Lawder HJ, Hara A, Kujak J, Pavlicek W (2010) Innovations in CT dose reduction strategy: application of the adaptive statistical iterative reconstruction algorithm. AJR Am J Roentgenol 194:191-199

6. Hara AK, Paden RG, Silva AC, Kujak JL, Lawder HJ, Pavlicek W (2009) Iterative reconstruction technique for reducing body radiation dose at CT: feasibility study. AJR Am J Roentgenol 193:764-771

7. Kilic K, Erbas G, Guryildirim M, Arac M, Ilgit E, Coskun B (2011) Lowering the dose in head $\mathrm{CT}$ using adaptive statistical iterative reconstruction. AJNR Am J Neuroradiol 32:1578-1582

8. Rapalino O, Kamalian S, Kamalian S et al (2012) Cranial CT with adaptive statistical iterative reconstruction: improved image quality with concomitant radiation dose reduction. AJNR Am J Neuroradiol 33:609-615

9. Komlosi P, Zhang Y, Leiva-Salinas C et al (2014) Adaptive statistical iterative reconstruction reduces patient radiation dose in neuroradiology CT studies. Neuroradiology 56:187-193

10. Vorona GA, Zuccoli G, Sutcavage T, Clayton BL, Ceschin RC, Panigrahy A (2013) The use of adaptive statistical iterative reconstruction in pediatric head CT: a feasibility study. AJNR Am J Neuroradiol 34:205-211

11. European Commission. EUR 16262 EN (2000) European guidelines for quality criteria for computed tomography. European Commission, Luxembourg

12. R Core Team (2013) R: A language and environment for statistical computing. R Foundation for Statistical Computing, Vienna

13. Hsieh J, Nett B, Yu Z, Sauer K, Thibault JB, Bouman CA (2013) Recent Advances in CT Image Reconstruction. Curr Radiol Rep 1: $39-51$

14. Rozeik C, Kotterer O, Preiss J, Schütz M, Dingler W, Deininger HK (1991) Cranial CT artifacts and gantry angulation. J Comput Assist Tomogr 15:381-386

15. Alberico RA, Loud P, Pollina J, Greco W, Patel M, Klufas R (2000) Thick-section reformatting of thinly collimated helical CT for reduction of skull base-related artifacts. AJR Am J Roentgenol 175:1361-6

16. American Association of Physicists in Medicine. CT scan protocols (2012) Routine Adult Head CT http://www.aapm.org/pubs/ CTProtocols/documents/AdultRoutineHeadCT.pdf. Accessed 03. 01.2015

17. Ren Q, Dewan SK, Li M (2012) Comparison of adaptive statistical iterative and filtered back projection reconstruction techniques in brain CT. Eur J Radiol 81:2597-2601

18. Kalender WA (2005) Computed tomography: fundamentals, system technology, image quality, applications. 2nd revised ed. Publicis Corporate, Erlangen

19. Schilham A, van der Molen AJ, Prokop M, de Jong HW (2010) Overranging at multisection CT: an underestimated source of excess radiation exposure. Radiographics 30:1057-1067

20. van der Molen AJ, Geleijns J (2007) Overranging in multisection CT: quantification and relative contribution to dose: comparison of four 16-section CT scanners. Radiology 242:208-216

21. Löve A, Olsson ML, Siemund R, Stålhammar F, BjörkmanBurtscher IM, Söderberg M (2013) Six iterative reconstruction algorithms in brain CT: a phantom study on image quality at different radiation dose levels. Br J Radiol 86:1031-1040

22. Schindera ST, Odedra D, Raza SA (2013) Iterative reconstruction algorithm for CT: can radiation dose be decreased while lowcontrast detectability is preserved? Radiology 269:511-518

23. Brady SL, Moore BM, Yee BS, Kaufman RA (2014) Pediatric CT: implementation of ASiR for substantial radiation dose reduction while maintaining pre-ASiR image noise. Radiology 270:223-231

24. Willemink MJ, Takx RA, de Jong PA et al (2014) Computed tomography radiation dose reduction: effect of different iterative reconstruction algorithms on image quality. J Comput Assist Tomogr 38:815-823 